\title{
Defined Study Subject Milestone
}

National Cancer Institute

\section{Source}

National Cancer Institute. Defined Study Subject Milestone. NCI Thesaurus. Code C93377.

An administrative activity defined at a global library level that represents a common administrative landmark for a study subject in the course of a study. 